\title{
Previsão das dimensões da zona termicamente afetada de juntas soldadas com variação de pa- râmetros de soldagem
}

\author{
Title: Welded Joints' Heat Affected Zone's Extension Prediction By Switching Weld- \\ ing Parameters
}

Leonardo G. T. Costa de Melo

Escola Politécnica de Pernambuco

Universidade de Pernambuco

50.720-001 - Recife, Brasil

lgtmelo@terra.com.br

\author{
Francisco Ilo Bezerra Cardoso \\ Escola Politécnica de Pernambuco \\ Universidade de Pernambuco \\ 50.720-001 - Recife, Brasil \\ ilo@poli.br
}

\author{
Ricardo Artur Sanguinetti \\ Departamento de Engenharia Mecânica \\ Universidade Federal de Pernambuco \\ 50.740-560 - Recife, Brasil \\ ras@ufpe.br
}

\begin{abstract}
Resumo A escolha de parâmetros de soldagem é uma etapa importante do processo de soldagem, influenciando diretamente no aporte térmico fornecido às juntas soldadas. Esse valor de aporte térmico juntamente com a distribuição de temperatura nas juntas soldadas fornece ao projetista condições de prever o tamanho da zona termicamente afetada, o tipo de microestrutura formada e consequentemente prevenir os efeitos das tensões residuais. Neste estudo foram variados três parâmetros que nos forneceram condições de soldagens diferentes. Cada condição foi analisada pelo software SmartWeld 2011 e por macrografias para encontrar e comparar o tamanho da zona termicamente afetada (ZTA). Ainda, foram calculadas as tensões residuais através do método de Deslocamento por Pontos Coordenados (DPC). É possivel escolher os melhores parâmetros para a junta de topo soldada pelo processo GMAW considerando os parâmetros em questão.
\end{abstract}

Palavras-Chave: tensão residual, parâmetros de soldagem, zta, deslocamento de pontos coordenados, chapas navais, gmaw.

\begin{abstract}
Choosing welding parameters is an important step in welding process, influencing directly in heat input provided to welded joints. This heat input value, along with temperature distribution in welded joints, provides to the draftsman conditions of predicting the Heat Affected Zone (HAZ) extension, the kind of microstructure to be formed, and therefore, predict the effects of residual stress. Three welding parameters were switched which provided different welding conditions. Each condition was analyzed by SmartWeld 2011 software and macrographs to find and compare the extension of HAZ, and the residual stresses, calculated through Displacement of Coordinated Points (DCP) method. It is possible to choose the best parameters for the welded joint by GMAW process considering the parameters in study.
\end{abstract}

Keywords: residual stress, welding parameters, haz, displacement of coordinated points, naval sheets, gmaw. 


\section{Introdução}

Em qualquer processo de fabricação mecânico surgem preocupações que devem ser levadas em conta. Podem ser a respeito de acabamento, dimensões, propriedades químicas, elétricas, ou, como é mais comum nos processos envolvendo soldagem, as propriedades mecânicas. No que diz respeito às últimas, as propriedades do metal de adição em si são passíveis de se controlar através do uso de consumíveis de ligas designadas favoravelmente, mas as Zonas Termicamente Afetadas (ZTAs) pelo calor da solda são frequentemente as regiões mais críticas no que se refere a potenciais falhas [1]. A ZTA determina as mudanças microestruturais e metalúrgicas na solda devido ao calor gerado durante o processo de soldagem e, normalmente, devido à microestrutura de grãos grosseiros, a ductilidade e tenacidade nesta área são baixas, sendo o fator mais importante dentre os que afetam a ZTA, o aporte térmico [2].

Ainda, em função da influência térmica, um material, elemento mecânico, ou peça acabada pode, ao fim do processo em questão, permanecer com tensões internas. Estas são denominadas tensões residuais: tensões auto equilibradas existentes nos materiais em condições de temperatura homogênea e sem carregamento externo [3]. Quando se lida com elevadas variações térmicas, tais tensões são introduzidas pelo elevado gradiente de temperatura devido ao aquecimento e resfriamento não homogêneo, e são originadas principalmente devido à contração no resfriamento, contribuindo também o resfriamento superficial intenso e as mudanças de fase. O material utilizado nesta pesquisa é um aço termicamente tratável, de modo que é possível afirmar que o fluxo de calor e o gradiente térmico durante a soldagem afetam a transformação de fase, modificando ponto a ponto a microestrutura e as propriedades da área afetada, sendo esse gradiente o grande responsável pela existência de tensões residuais e distorções.

Nas amostras analisadas foram variados a tensão de soldagem, a velocidade de soldagem, e o ângulo de bisel. Contudo, para facilitar as comparações e posteriores análises, apenas um único parâmetro foi alterado por amostra, enquanto que os demais permaneceram constantes. Para o caso da variação no ângulo de bisel, permaneceram constantes os demais parâmetros de soldagem, de modo que o aporte térmico não variasse. Este pode ser calculado através da equação 1 a seguir [4].

$$
E=\frac{\eta \times \mathrm{V} \times \mathrm{I}}{v}
$$

Sendo: $\eta$ - eficiência de transferência, a depender do processo;

$V$ - tensão em volts (V);

I - corrente elétrica em ampere (A);

$v$ - velocidade linear de soldagem, em $\mathrm{mm} / \mathrm{s}$.

$\mathrm{O}$ aporte térmico é uma característica importante, pois assim como as temperaturas de preaquecimento e interpasses, influencia a taxa de resfriamento, que afeta as propriedades mecânicas e estrutura metalúrgica das regiões soldada e afetada pelo calor [5] e, consequentemente, afeta a extensão da ZTA e a distribuição de tensões residuais na soldagem.

\section{Materiais e Métodos}

\subsection{Metal de base}

As chapas utilizadas no trabalho foram fornecidas pelo Estaleiro Atlântico Sul, localizado no Porto de Suape Cabo de Santo Agostinho - PE, com as dimensões de 1200 x 500 x 13,7 mm e classificação ASTM A131 grau AH-36.

Para as chapas de teste serem soldadas foi necessária a preparação das mesmas, sendo preciso cortá-las nas dimensões de $70 \times 200 \times 13,7 \mathrm{~mm}$ com o sentido de laminação longitudinal, para que os cordões de solda fossem produzidos paralelamente à direção de laminação.

\subsection{Equipamentos, processo e parâmetros de soldagem}

Foram utilizadas chapas de aço naval ASTM A-131 grau AH-36 com o objetivo de se calcular tensões residuais através do método de Deslocamento de Pontos Coordenados (DPC) e posteriormente, extrair amostras para avaliar as extensões das ZTAs. As chapas foram usinadas como demonstra a Figura 1, com altura de raiz de $2 \mathrm{~mm}$ e ângulo de bisel de $25^{\circ}$, ou ainda, $35^{\circ}$.

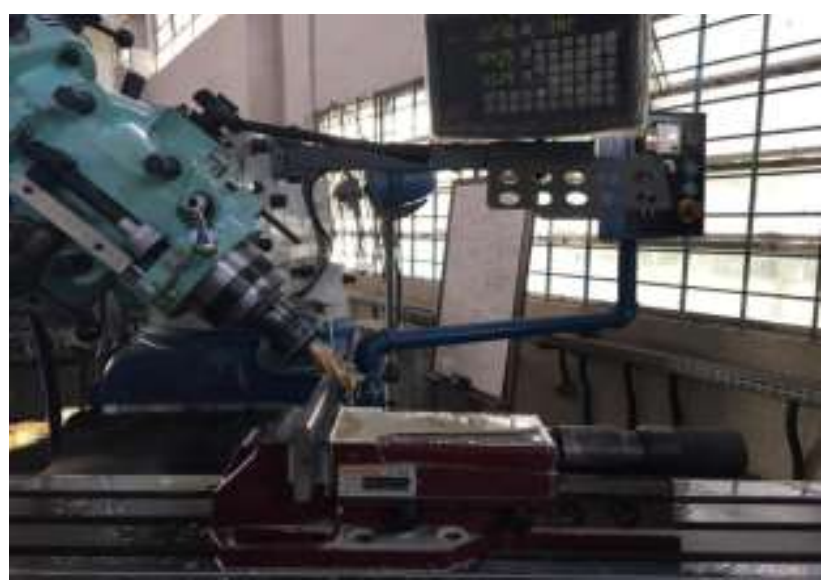

Figura 1: Chapa em processo de usinagem. 
Foram realizadas soldagens através do processo GMAW com uma fonte semiautomática de soldagem MAG, como demonstra a Figura 2, em conjunto com uma máquina de corte a gás adaptada para realizar o translado da pistola (Figura 3), automatizando a operação.

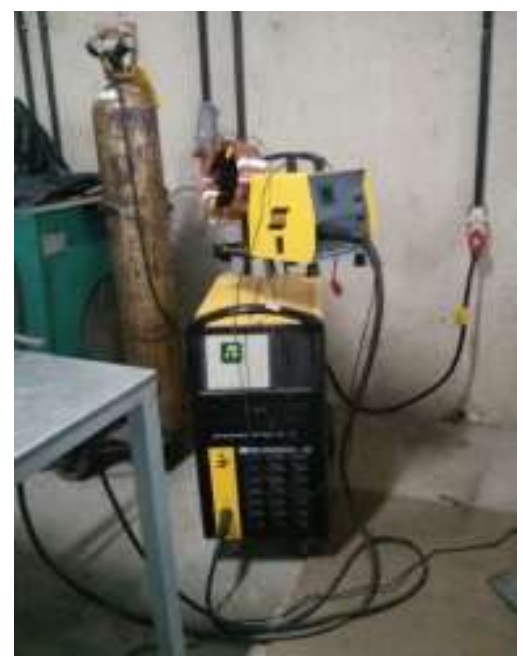

Figura 2: Máquina de soldagem MAG semiautomática.

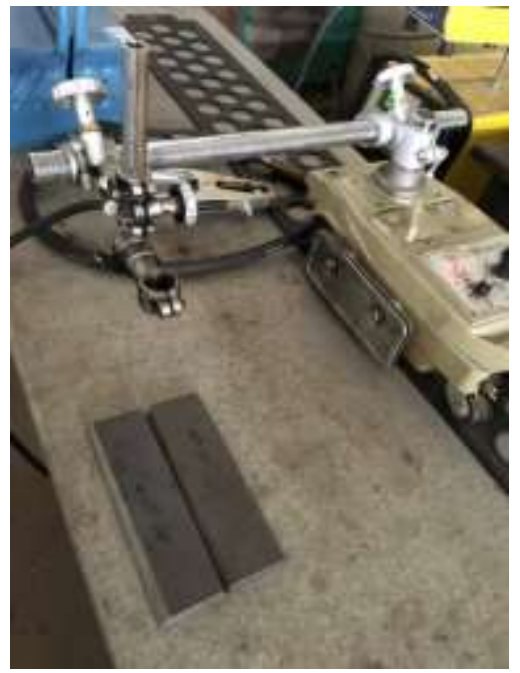

Figura 3: Máquina de corte a gás.

O arame utilizado foi o AWS ER70S-6 com 1,2 mm de diâmetro e o gás, uma mistura de $75 \%$ de $\operatorname{Ar}$ e $25 \%$ de $\mathrm{CO} 2$. Para simular as soldagens de painéis navais, utilizaram-se travas laterais como maneira de impor restrições. A máquina de corte a gás foi então alinhada para o passe de raiz, e os passes subsequentes. Os passes foram realizados sempre se alternando o sentido para minimizar distorções. Após cada passe, o filme vítreo formado foi removido com uma escova de aço.

Quatro pares de chapas de $70 \times 200 \mathrm{~mm}$ foram soldadas duas a duas com os parâmetros listados na Tabela 1 abaixo. Tomando a amostra 1 como referencial, para as demais amostras foi variado apenas um parâmetro, de modo que fosse possível a comparação em função de cada alteração. Os parâmetros variados foram o ângulo de bisel, para a amostra 2, a velocidade de soldagem, para a amostra 3, e a tensão média, para a amostra 4 .

\begin{tabular}{|c|c|c|c|c|c|}
\hline Amostra & $\begin{array}{c}\text { Tensão } \\
\text { média } \\
\text { (V) }\end{array}$ & $\begin{array}{l}\text { Corrente } \\
\text { média } \\
\text { (A) }\end{array}$ & $\begin{array}{c}\text { Velocidade } \\
\text { de soldagem } \\
(\mathrm{mm} / \mathrm{s})\end{array}$ & $\begin{array}{c}\text { Aporte } \\
\text { médio } \\
\text { (W) }\end{array}$ & g bisel $\left({ }^{\circ}\right)$ \\
\hline 1 & 19,53 & 173,67 & 6 & 565,38 & 25 \\
\hline 2 & 19,99 & 169,33 & 6 & 564,13 & 35 \\
\hline 3 & 19,49 & 174,75 & 3,5 & 972,98 & 25 \\
\hline 4 & 26,91 & 178,42 & 6 & 800,15 & 25 \\
\hline
\end{tabular}

Tabela 1: Parâmetros de soldagem utilizados

\subsection{Medições de tensões residuais}

O método utilizado para medir as tensões residuais foi o DPC [6], que consiste em se observar o deslocamento de pontos da chapa. Para tal, foram realizados cinco furos com $2 \mathrm{~mm}$ de profundidade usando uma broca de $2 \mathrm{~mm}$ de diâmetro (Figura 4), cujos centros foram então mapeados em (X, Y).

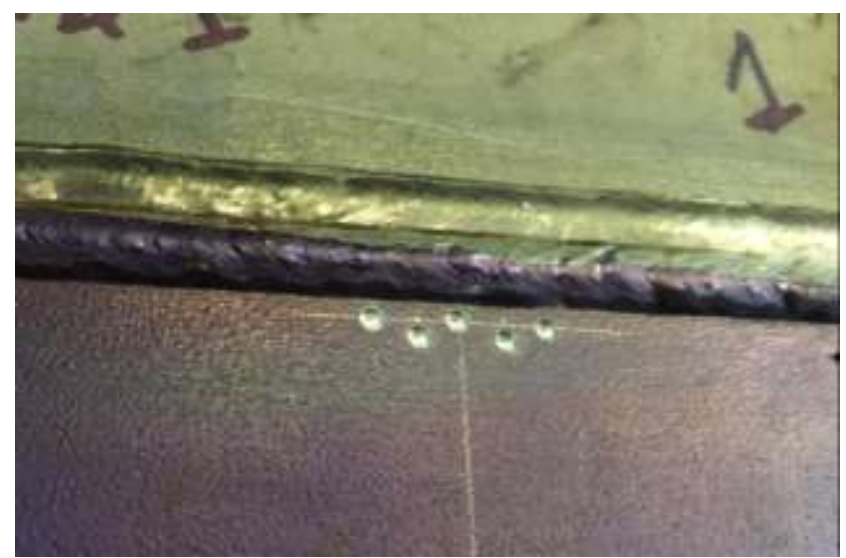

Figura 4: Furos realizados na amostra.

A distribuição dos furos é dada conforme a ilustração a seguir (Figura 5).

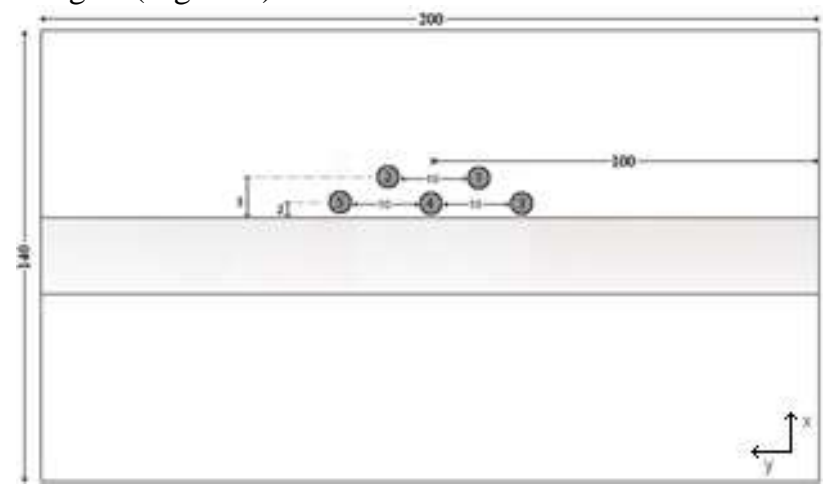

Figura 5: Esquema de distribuição de furos nas chapas soldadas. 
A medição e mapeamento dos furos é realizada com uma Máquina de Medição de Coordenadas (MMC) com controle numérico computadorizado (Figura 6).

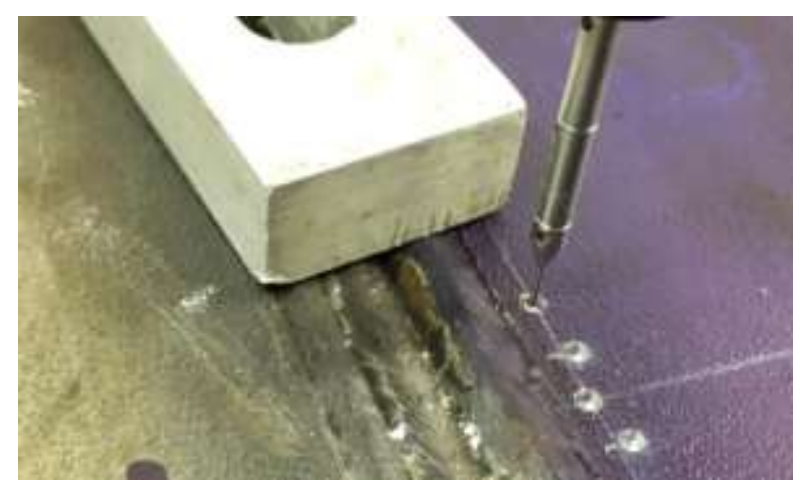

Figura 6: Medição sendo realizada na MMC.

A próxima etapa consiste na realização de um tratamento térmico de recozimento (Figura 7), o método mais utilizado atualmente para aliviar as tensões residuais [4], a $740^{\circ} \mathrm{C}$ com o objetivo de aliviar as tensões, sendo assim possível a identificação dos deslocamentos dos pontos medidos, uma vez que houve a reversão da plastificação. Essa condição acontece pois durante o tratamento térmico, a tensão de recuo atua revertendo as barreiras, contribuindo para o deslocamento (escoamento) na direção contrária à plastificação. [7]

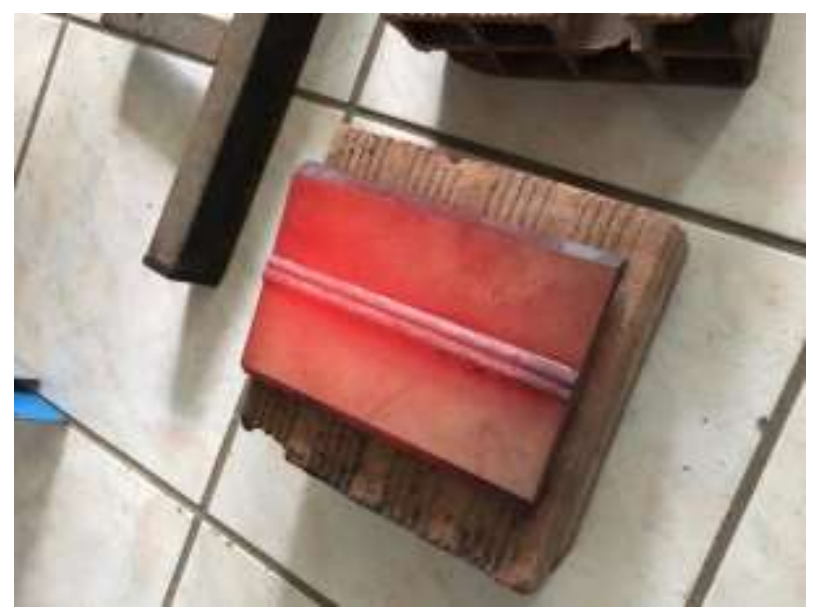

Figura 7: Chapa retirada do forno após tratamento térmico.

Os pontos mapeados sofreram deslocamentos devido ao escoamento reverso do material e foram remapeados, de acordo com a técnica de medição de coordenadas, que determina os parâmetros dimensionais através da medição das coordenadas de pontos sobre a superfície de uma peça e os processa matematicamente [8]. Com isso, é possível calcular a deformação através da equação 2 , tanto no sentido longitudinal quanto transversal.

$$
\varepsilon=\frac{\Delta L}{L_{0}}
$$

Sendo:

$\varepsilon$ : deformação específica;

$\Delta \mathrm{L}$ : distância dos pontos coordenados (mm);

L0: distância entre o ponto e o centro da chapa ( $\mathrm{mm})$.

Com as coordenadas dos pontos medidos antes e após o tratamento térmico, as tensões de soldagem podem ser calculadas através das equações (3) e (4) [9]:

$$
\begin{aligned}
& \sigma_{x}=\frac{E}{1-v^{2}}\left(\varepsilon_{x}+v \varepsilon_{y}\right) \\
& \sigma_{y}=\frac{E}{1-v^{2}}\left(\varepsilon_{y}+v \varepsilon_{x}\right)
\end{aligned}
$$

Sendo:

$\sigma_{\mathrm{x}}$ : tensão residual longitudinal - direção de soldagem $(\mathrm{Pa})$;

$\sigma_{\mathrm{y}}$ : tensão residual transversal - normal à direção de soldagem $(\mathrm{Pa})$;

$\varepsilon_{\mathrm{x}}$ : deformação específica paralela ao cordão de solda;

$\varepsilon_{\mathrm{y}}$ : deformação específica perpendicular ao cordão de solda;

E: módulo de elasticidade do material (GPa);

$v$ : coeficiente de Poisson.

Ainda, os valores de tensões residuais $\sigma_{x}$ e $\sigma_{y}$ são obtidos pela medição de $\varepsilon_{\mathrm{x}}$ e $\varepsilon_{\mathrm{y}}$, que medem as extensões dos deslocamentos reversos nos pontos onde se deseja conhecer a tensão residual [9]. Para calculá-los, adotou-se o módulo de elasticidade de $207 \mathrm{GPa}$ e coeficiente de Poisson de 0,3 [7].

\subsection{Medição e modelagem da ZTA}

Como as soldagens realizadas foram multipasses, para medição efetiva da extensão da ZTA relativa ao processo foi realizada a identificação do último passe de solda. Para tal, foram realizadas as macrografias abaixo (Figuras 8-11) em corpos de prova extraídos de cada amostra.

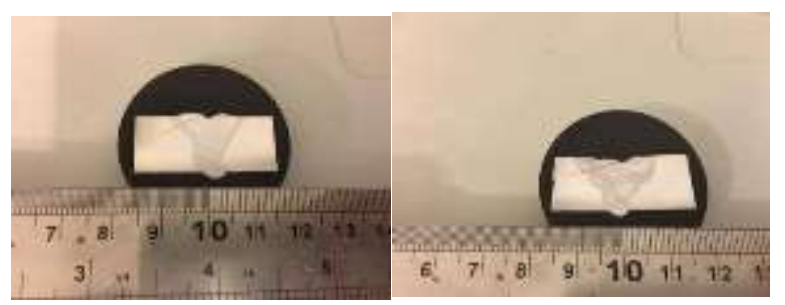

Figuras 8 e 9: Macrografias dos CPs das amostras 1 e 2. 


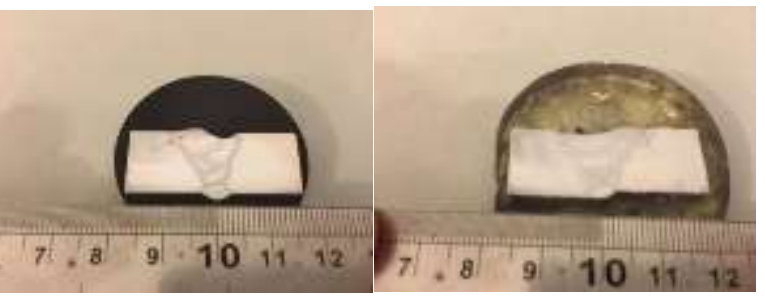

Figura 10 e 11: Macrografias dos CPs das amostras 3 e 4

A medição foi realizada com o uso do instrumento de medição dimensional de um microscópio ótico nas regiões identificadas nas figuras.

Para fins comparativos, foi realizada a modelagem do cordão de solda através do software SmartWeld 2011, com o uso do módulo ISO 2.5D. Por meio desta simulação foram obtidas representações do gradiente das temperaturas alcançadas no plano transversal ao cordão de solda, sendo apenas necessário preencher qual temperatura deseja-se visualizar na representação. Para a realização das simulações faz-se necessário escolher um material de um banco de dados próprio e não editável do software. Como não consta o aço ASTM A131 grau AH-36, selecionamos o aço AISI 1018 por ser o que mais se assemelha dentre os ofertados. O SmartWeld não possui suporte a soldagens multipasses, sendo modelado apenas um filete de solda. Como o que está em estudo efetivamente é a ZTA relativa ao último passe de solda, isso não foi um problema. A temperatura de recristalização do aço utilizado nesta pesquisa é de $750^{\circ} \mathrm{C}$ [10], sendo este o valor adotado para a identificação da isotérmica correspondente ao início da ZTA.

\subsection{Resultados e discussão}

Foram calculadas as tensões na direção longitudinal e perpendicular ao cordão de solda para cada um dos cinco pontos de cada amostra. Os módulos das tensões foram então agrupados de acordo com a distância entre o ponto em questão e o cordão de solda. Assim, foram agrupados os pontos 1 e 2, cujos centros distanciam-se $3 \mathrm{~mm}$ da margem do cordão de solda, e 3, 4 e 5, com centros localizados a $2 \mathrm{~mm}$ da margem do cordão de solda. A média dos valores dos pontos agrupados foi então compilada na Tabela 2 .

\begin{tabular}{ccccc}
\hline Amostra & $\begin{array}{c}|\boldsymbol{\sigma x \mathbf { l } , 2 \mathbf { a }}| \\
(\mathbf{M P a})\end{array}$ & $\begin{array}{c}|\boldsymbol{\sigma x \mathbf { 3 } , 4 , 5 a}| \\
(\mathbf{M P a})\end{array}$ & $\begin{array}{c}|\boldsymbol{\sigma y} \mathbf{l}, \mathbf{2 a}| \\
(\mathbf{M P a})\end{array}$ & $\begin{array}{c}|\boldsymbol{\sigma y} \mathbf{3 , 4 , 5 a}| \\
(\mathbf{M P a})\end{array}$ \\
\hline $\mathbf{l}$ & 184,18 & 202,96 & 232,29 & 252,45 \\
\hline $\mathbf{2}$ & 92,08 & 104,34 & 109,56 & 123,68 \\
\hline $\mathbf{3}$ & 18,12 & 14,26 & 25,82 & 19,72 \\
\hline $\mathbf{4}$ & 83,49 & 80,51 & 104,16 & 102,83 \\
\hline
\end{tabular}

Tabela 2: Tensões residuais.

Os resultados encontrados estão condizentes com os intervalos de valores de tensões residuais encontrados em chapas com espessuras similares [11]. É possível observar a redução nas tensões residuais quando se eleva o ângulo de bisel (no caso, de $25^{\circ}$ a $35^{\circ}$ ), com demanda de mais passes e consequente aumento de volume de solda depositada. De forma análoga, ocorre redução nas tensões residuais com a redução da velocidade, bem como com o aumento da tensão elétrica, uma vez que ambos os casos implicam em aumento do aporte térmico. $\mathrm{O}$ aporte térmico e o número de passes tem grande efeito na distribuição das tensões residuais: com o aumento do aporte térmico e do número de passes, as tensões residuais decrescem, sendo a soldagem multipasses e o elevado aporte térmico úteis para redução nas tensões residuais [12].

A Tabela 3 lista os valores das medições da extensão das ZTAs obtidas experimentalmente, a partir de cada amostra embutida.

\begin{tabular}{cc}
\hline Amostra & Largura da ZTA (mm) \\
\hline $\mathbf{1}$ & 1,80 \\
\hline $\mathbf{2}$ & 1,60 \\
\hline $\mathbf{3}$ & 2,10 \\
\hline $\mathbf{4}$ & 2,00 \\
\hline
\end{tabular}

Tabela 3: Larguras das ZTAs obtidas experimentalmente

As imagens a seguir (Figuras 12-15) foram os resultados obtidos nas modelagens realizadas no SmartWeld.

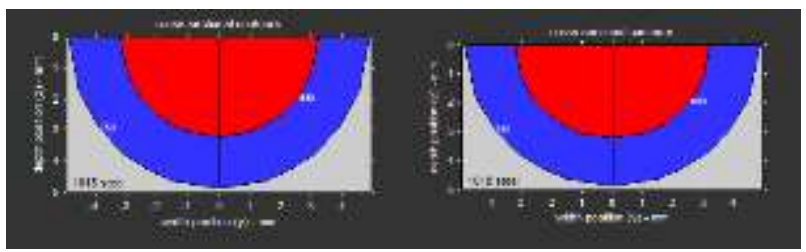

Figuras 12 e 13: Modelagens das amostras 1 e 2, respectivamente.

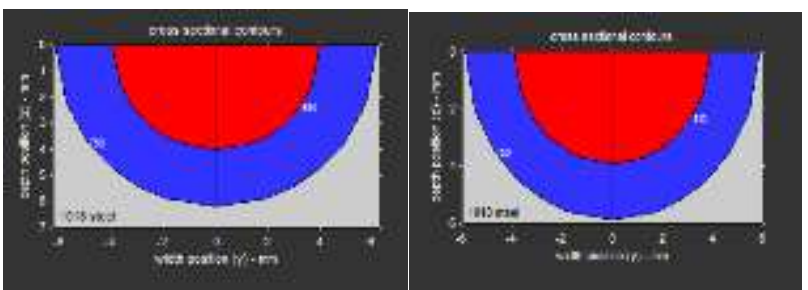

Figuras 14 e 15: Modelagens das amostras 3 e 4, respectivamente.

A partir as imagens obtidas pelo modelo, foram obtidos os valores das extensões das ZTAs, mostrados na Tabela 4.

\begin{tabular}{cc}
\hline Amostra & Largura da ZTA (mm) \\
\hline $\mathbf{1}$ & 1,8 \\
\hline $\mathbf{2}$ & 1,8 \\
\hline $\mathbf{3}$ & 2,1 \\
\hline $\mathbf{4}$ & 2,0 \\
\hline
\end{tabular}

Tabela 4: Larguras das ZTAs segundo SwartWeld. 
A partir dos valores obtidos com as medições e simulações, observa-se que o SmartWeld é uma ferramenta precisa para usos equivalentes ao do estudo em questão, principalmente ao se considerar os poucos parâmetros que exige para realizar uma modelagem como esta. Três das amostras medidas $\left(\mathrm{n}^{\circ} 1,3\right.$ e 4$)$ apresentaram o mesmo valor de largura da ZTA que o previsto pelo software, enquanto que a única amostra com divergência $\left(\mathrm{n}^{\circ} 2\right)$ apresenta um erro baixo, considerando as aproximações e adequações realizadas. Ainda, o software não aceita a configuração do ângulo de bisel como um parâmetro.

Se levarmos em conta unicamente o valor de tensões residuais menores devemos escolher velocidades de soldagens menores, mas teremos tamanhos de ZTA maiores. Entretanto, se levarmos em conta unicamente o tamanho da ZTA, escolheremos trabalhar com ângulos de bisel maiores, apesar da maior demanda de volume de solda para preenchimento efetivo, que na prática, limitaria o parâmetro. Porém, teremos as tensões reduzidas pela metade. As mudanças de parâmetros de soldagem afetam os valores de tensões residuais para mais e o tamanho da ZTA para menos, e vice-versa, então cabe ao engenheiro escolher o que precisa ser levado em conta na hora de escolher os parâmetros de soldagem.

\section{Conclusões}

O software SmartWeld mostrou-se uma ferramenta razoável para predizer a extensão da ZTA para um único passe de soldagem, apresentando valores coerentes com os medidos nas amostras $n^{\circ} 1,3$ e 4 . A divergência na amostra $\mathrm{n}^{\circ} 2$, com variação do ângulo de bisel, pode ser atribuída ao fato do software não aceitar a configuração de ângulo de bisel como um parâmetro.

Ao analisar a mudança de bisel de $25^{\circ}$ para $35^{\circ}$, as tensões residuais foram reduzidas pela metade, como esperado. Para a mudança de velocidade de $6 \mathrm{~mm} / \mathrm{s}$ para $3,5 \mathrm{~mm} / \mathrm{s}$, o tamanho da ZTA aumentou em $0,3 \mathrm{~mm}$ e as tensões residuais diminuíram entre 160 e $230 \mathrm{MPa}$. Mostrando que podemos ter um tamanho de ZTA maior, porém reduzimos significativamente as tensões residuais.

Para a mudança da tensão de soldagem de $19,53 \mathrm{~V}$ para $26,91 \mathrm{~V}$ o tamanho da ZTA aumentou em $0,2 \mathrm{~mm}$ e as tensões residuais diminuíram um pouco mais que a metade. Mais uma vez, mostrando que podemos ter uma ZTA maior, mas teremos tensões residuais menores.

\section{Referências}

[1] B. Hutchinson, J. Komenda, G. S. Rohrer, H. Beladi. Heat affected zone microstructures and their influence on toughness in two microalloyed
HSLA steels. Acta Materialia 97, 2015.

[2] M. A. Moghaddam, R. Golmezergi, F. Kolahan. Multi-variable measurements and optimization of GMAW parameters for API-X42 steel alloy using a hybrid BPNN-PSO approach. Measurement 92, p. 279-287, 2016.

[3] E. Macherauch,K. H. Kloss. "Origin, Measurements and Evaluation of Residual Stress in Science and Technology". Ed. by Macherauch, V. Hauk, DGM VERLAG, 1997.

[4] P. J. Modenesi. Efeitos Mecanicos do Ciclo Térmico. Departamento de Engenharia Metalúrgica e de Materiais. Universidade Federal de Minas Gerais - UFMG. 2001

[5] W. Zinn, B. Scholtes. Residual Stress Formation Processes During Welding and Joining, Handbook of Residual Stress and Deformation of Steel, ASM Inter., p. 391-396, 2002.

[6] A. V. Siqueira Filho. Estudo Comparativo das Tensões Residuais em Juntas Soldadas pelas Técnicas de Medição por Coordenadas e Difração de Raios-X. Tese de Doutorado. Universidade Federal de Pernambuco, Fev 2012.

[7] C. E. Mendes. Previsão de tensões residuais em juntas soldadas de painéis navais pelo método DPC. Dissertação de Mestrado, Universidade federal de Pernambuco. Out 2015.

[8] T. L. Rolim. Sistemática indicadora de método para calibração de máquinas de medição por coordenadas. Tese de Doutorado. Universidade Federal da Paraíba. Dez 2003.

[9] T. Okumura, C. Tanigusgi. Engenharia de soldagem e aplicações. Livros Técnicos e Científicos Editora, Rio de Janeiro, 1982.

[10] I. L. Gonçalves. Influência da Temperatura no Tratamento de Alívios de Tensões em uma Junta Soldada Baseado no Método DPC. Dissertação de Mestrado, UFPE. Jul 2015.

[11] W. C Jiang, B. Y. Wang, J. M. Gong, S. T. Tu. Finite element analysis of the effect of welding heat input and layer number on residual stress in repairwelds for a stainless steel clad plate. Materials \& Design 32, p. 2851-2857, 2011.

[12] T. J. Kim, B. S. Jang, S.W. Kang. Welding deformation analysis based on improved equivalent strain method considering the effect of temperature gradients. International Journal of Naval Architecture and Ocean Engineering, v. 7, 2015. 